\title{
Semiconductor Sensor for Hydrogen Sulfide on the Basis of Tungsten and Copper Oxides
}

\author{
Abdurakhmanov Ilxom Eergashboyevich ${ }^{1}$, Begmatov Rizamat Khushvaqtovich ${ }^{2}$, \\ Abdurakhmanov Ergashboy ${ }^{2}$, Kholboev Orif Nurmatovich ${ }^{3}$ \\ ${ }^{1}$ Department of Inorganic Chemistry and Materials Science, Samarkand State University, Samarkand, Uzbekistan \\ ${ }^{2}$ Department of Analytical Chemistry, Samarkand State University, Samarkand, Uzbekistan \\ ${ }^{3}$ Djizzakh State Pedagogical Institute, Djizakh, Uzbekistan
}

\section{Email address:}

ergash50@yandex.ru (A. I. Eergashboyevich), ergash50@yandex.ru (B. R. Khushvaqtovich), ergash50@yandex.ru (A. Ergashboy), ergash50@yandex.ru (K. O. Nurmatovich)

\section{To cite this article:}

Abdurakhmanov Ilxom Eergashboyevich, Begmatov Rizamat Khushvaqtovich, Abdurakhmanov Ergashboy, Kholboev Orif Nurmatovich. Semiconductor Sensor for Hydrogen Sulfide on the Basis of Tungsten and Copper Oxides. International Journal of Management and Fuzzy Systems. Vol. 6, No. 4, 2020, pp. 72-79. doi: 10.11648/j.ijmfs.20200604.13

Received: March 5, 2020; Accepted: March 20, 2020; Published: December 31, 2020

\begin{abstract}
Hydrogen sulfide is one of the main toxic and explosive components of atmospheric air, found in the waters of mineral springs, volcanic gases, and petroleum and natural gas fields. It is used in the production of sulfur, sulfuric acid, mineral fertilizers, sulfides, organosulfur compounds, in analytical chemistry and medicine. $\mathrm{H}_{2} \mathrm{~S}$ is an active poisonous substance of neuroparalytic action. Upon inhalation of $1.0 \mathrm{mg} / \mathrm{l}$ and higher hydrogen sulfide, poisoning with it can develop almost instantly: convulsions and loss of consciousness end in quick death from respiratory and cardiac attack. The maximum permissible concentration of hydrogen sulfide in the air of production zones is $10 \mathrm{mg} / \mathrm{m}^{3}$. The presence of hydrogen sulfide in environmental objects is a serious danger, both from an environmental and technical point of view. Its presence causes chemical and electrochemical corrosion, is easily ignited $\left(\right.$ at $\left.346^{\circ} \mathrm{C}\right)$, and in a mixture with air explodes. Explosive concentrations in the mixture with air are at the level of 4.3-45.5\%. With regards above mentioned the control of pre-explosion and fire-hazardous concentrations of hydrogen sulfide is one of the most important tasks of safety and solving various fire-fighting measures. A method for increasing the selectivity and sensitivity of semiconductor methods for determining hydrogen sulfide is proposed, based on the use of optimal values of the sensor temperature and the composition of the gas-sensitive material of catalysts with inadequate activity to different components of the gas mixture. As a result of the conducted experiments, a selective semiconductor hydrogen sulfide sensor was developed, which provides rapid determination of hydrogen sulfide in a wide range of its concentration in atmospheric air and process gases. The main metrological characteristics and operational parameters of the developed selective semiconductor sensors for the determination of hydrogen sulfide are evaluated. Selectivity, measurement range and basic errors, additional sensor errors due to changes in ambient temperature and pressure are studied. The change in the flow rate of the gas mixture in the studied interval $(5-50 \mathrm{l} / \mathrm{h})$ does not significantly affect the value of the sensor output signal. Scope of the developed sensors: environmental monitoring of atmospheric air and process gases. The developed semiconductor hydrogen sulfide sensors are not inferior in accuracy and reproducibility to well-known foreign analogues, while retaining the following characteristics: expressiveness, portability, ease of manufacture and operation. The value of the relative standard deviation $\left(\mathrm{S}_{\mathrm{r}}\right)$ due to immutable components does not exceed 0.05 . The output signal of the sensors also does not depend on the location in space and the angles of inclination, which makes it possible to classify the developed sensors (according to GOST-13320-82) as independent.
\end{abstract}

Keywords: Semiconductor, Metrological Characteristics, Sensitivity, Selectivity, Hydrogen Sulfide, Tungsten Oxide, Copper Oxide 


\section{Introduction}

With the rapid development of the chemical and petrochemical industry, environmental monitoring involves constant analytical control of the purity of atmospheric air from sulfur compounds (in particular from hydrogen sulfide) [1]. An original solution to the problem of monitoring atmospheric air is the use of selective sensors and automatic gas analyzers based on the semiconductor method $[2,3]$. The main advantages of the semiconductor method and sensors developed on its basis are ease of use, portability, a significant resource of work, high accuracy and speed, which makes it easy to automate the process and collect and accumulate the necessary analytical information [3].

Hydrogen sulfide is one of the main toxic and explosive components of atmospheric air, found in the waters of mineral springs, volcanic gases, oil and natural gas deposits. They are used in the production of sulfur, sulfuric acid, mineral fertilizers, sulfides, organosulfur compounds, in analytical chemistry and medicine. $\mathrm{H}_{2} \mathrm{~S}$ is an active poisonous substance of neuroparalytic action. After inhalation of $1.0 \mathrm{mg} / \mathrm{l}$ and higher, hydrogen sulfide poisoning can develop almost instantly: convulsions and loss of consciousness result in quick death from respiratory arrest and heart failure. The maximum permissible concentration of hydrogen sulfide in the air of production zones is $10 \mathrm{mg} / \mathrm{m}^{3}$ [4].

The presence of hydrogen sulfide in environmental objects is a serious danger, both from an environmental and technical point of view. Its presence causes chemical and electrochemical corrosion, easily ignites (at $346^{\circ} \mathrm{C}$ ), and explodes in a mixture with air [6, 7]. Explosive concentrations in a mixture with air are at the level of 4.3$45.5 \%$. In relation with the foregoing, the control of preexplosion and fire hazardous concentrations of hydrogen sulfide is one of the most important tasks of safety and the solution of various fire prevention activities.

\section{Experimental Sections}

\subsection{The Methods of the Experiment}

The principle of operation of semiconductor hydrogen sulfide sensors is to change the conductivity of the gassensitive material during chemical sorption of the detected gases on the surface of the semiconductor. Of particular interest in sensors are semiconductor gas-sensitive materials based on metal oxides. In this regard, we have studied the possibility of using these tungsten oxide and copper as a gas sensitive layer of selective semiconductor sensors for monitoring the content of hydrogen sulfide. The cheapest, most economical, and convenient method for obtaining a given structure of semiconductor gassensitive nanocomposites is the sol-gel method, which is widely used to obtain gels based on TEOS (silicon dioxide) $[8,9]$.
The sol-gel method is potentially capable of mixing components at the molecular level, creating structures with controlled porosity and controlled geometry of the gassensitive phase immobilized inside the inorganic polymer.

Therefore, experiments related to the study of the laws governing the formation of gas-sensitive thin films using the sol-gel technology and the development of highly sensitive, selective sensors and automatic gas analyzers of hydrogen sulfide on their basis seem to actual.

The field of application of semiconductor hydrogen sulfide sensors ("SC- $\mathrm{H}_{2} \mathrm{~S}$ ") covers many technological, medical and environmental tasks where constant monitoring of gas medium components is required. Gas sensors based on semiconductor elements attract considerable attention of specialists due to their extremely high sensitivity to the composition of the gas phase, simplicity of design and the ability to integrate into information systems. As a gassensitive material of the semiconductor layer for detecting micro-concentrations of gases, metal oxides are mainly used [10].

We have shown the possibility of using metal oxides of $\mathrm{W}$ and $\mathrm{Cu}$ as a gas sensitive material for the semiconductor hydrogen sulfide sensor $[11,12]$.

Sensitive elements of the sensors were fabricated using a method involving the deposition of $\mathrm{SiO}_{2}-\mathrm{WO}_{3}$ sols onto a spiral of vitrified platinum wire, followed by their thermal dehydration and processing at $550^{\circ} \mathrm{C}$. This method makes it possible to obtain oxide films with a given phase composition and surface morphology, and also provides a uniform distribution of modifying additives in them. A thin gas-sensitive film and a catalyst (copper oxide) were deposited onto the surface by the sol-gel method. The sols used to produce gas-sensitive materials and a catalyst were synthesized from tetroethoxysilane and salts of the corresponding metals. A platinum microwire located inside the glass tube acts as a heater.

Developed sensors $\left(\mathrm{SC}-\mathrm{H}_{2} \mathrm{~S}\right)$ provide monitoring of hydrogen sulfide concentrations in a gas mixture. The sensor characteristics of the systems under consideration in the process of determining hydrogen sulfide were studied using standard methods.

The sensitivity of the sensor (S) was determined by the formula:

$$
\mathrm{S}=\mathrm{R}_{\mathrm{in}} / \mathrm{R}_{\mathrm{gas}}=\sigma_{\mathrm{gas}} / \sigma_{\text {in }}
$$

where $R_{\text {gas }}$ and $\sigma$ gas are the resistance and electrical conductivity of the film when exposed to hydrogen sulfide of a given concentration; $R_{\text {in }}$ and $\sigma_{\text {in }}$ are the resistance and electrical conductivity of the film in air in the absence of hydrogen sulfide (corresponds to the value of the conductivity of the film at zero gas concentration).

The test program in the operating mode included special experiments related to the establishment of dynamic, calibration characteristics, as well as the identification of the measurement range, the main errors, the degree of selectivity and stability of the semiconductor hydrogen sulfide sensor 


\section{"SC- $\mathrm{H}_{2} \mathrm{~S}$ ".}

\subsection{Dynamic Characteristics of the Hydrogen Sulfide Sensor $\mathrm{SC}-\mathrm{H}_{2} \mathrm{~S}$}

One of the main requirements for sensors of fire and explosive components is to ensure the rapid determination of the component, (i.e., a short transition time), established by the study of their dynamic characteristics.

Checking the dynamic characteristics of the developed hydrogen sulfide sensor includes the study of its temporal characteristics, that is, the time of transient processes. Before starting the test, it is necessary to set the value of the output (background) signal, for this, purified air was supplied through five sequentially connected semiconductor hydrogen sulfide sensors for 10 minutes and the signals (value of the gas-sensitive layer resistance) were recorded using a KSP-4 and a B7-35 digital voltmeter. In the experiments, Test gas mixture (TGM) with a hydrogen sulfide concentration of 100 $\mathrm{mg} / \mathrm{m}^{3}$ and $0.2 \%$ vol.

Checking the dynamic characteristics of the sensor was accompanied by continuous recording of the transient process of the chart tape of a recording device, the speed of which was chosen so that the graph of the transient process was adequate to GOST 133220-81 "Gas analyzers, industrial automatic." General technical parameters of the sensor fit on a piece of chart tape, $25 \mathrm{~cm}$ long.

The change in concentration at the input of the sensor was noted on the chart tape and was taken as the beginning of the countdown. The results of determining the dynamic characteristics of the $\mathrm{SC}-\mathrm{H}_{2} \mathrm{~S}$ semiconductor sensor are shown in Table 1.

Table 1. Dynamic characteristics of a semiconductor hydrogen sulfide sensor

\begin{tabular}{|c|c|c|c|c|c|c|c|}
\hline \multirow{2}{*}{ No } & \multirow{2}{*}{$\begin{array}{l}\text { Concentration } \mathrm{H}_{2} \mathrm{~S}, \mathrm{mg} / \mathrm{m} \\
\text { (vol. \%) }\end{array}$} & \multirow{2}{*}{$\begin{array}{l}\text { Dynamic characteristics of } \\
\text { the sensor* }\end{array}$} & \multicolumn{5}{|l|}{ Time } \\
\hline & & & SC-1 & SC-2 & $\mathrm{SC}-3$ & SC-4 & SC-5 \\
\hline \multicolumn{8}{|c|}{ Concentration $\mathrm{H}_{2} \mathrm{~S}, \mathrm{mg} / \mathrm{m}^{3}$} \\
\hline 1 & 100 & $\mathrm{t}_{0.1}$ & 1 & 2 & 1 & 1 & 2 \\
\hline 2 & 100 & $\mathrm{t}_{0.65}$ & 4 & 3 & 5 & 5 & 4 \\
\hline 3 & 100 & $\mathrm{t}_{0.9}$ & 7 & 7 & 7 & 8 & 8 \\
\hline 4 & 100 & $t_{t}$ & 9 & 10 & 10 & 10 & 9 \\
\hline \multicolumn{8}{|c|}{ Concentration $\mathrm{H}_{2} \mathrm{~S}$, vol\%. } \\
\hline 5 & 0.250 & $\mathrm{t}_{0.1}$ & 1 & 1 & 1 & 1 & 2 \\
\hline 6 & 0.250 & $\mathrm{t}_{0.65}$ & 4 & 4 & 5 & 4 & 4 \\
\hline 7 & 0.250 & $\mathrm{t}_{0.9}$ & 7 & 7 & 7 & 7 & 7 \\
\hline 8 & 0.250 & $t_{t}$ & 9 & 10 & 10 & 10 & 9 \\
\hline
\end{tabular}

* $\mathrm{t}_{0.1}$ - response start time, $\mathrm{s} ; \mathrm{t}_{0.65}$ - constant time, $\mathrm{s} ; \mathrm{t}_{0.9}$ - time of establishment of readout signal, $\mathrm{s} ; \mathrm{t}_{\mathrm{t}}$ is the sum of total measurement time, $\mathrm{s}$.

As can be seen from the data, the developed sensors have a response start time $\left(\mathrm{t}_{0.1}\right)$ of $1-2 \mathrm{~s}$, a constant time $\left(\mathrm{t}_{0.65}\right)$ of no more than $5 \mathrm{~s}$, and a readout time $\left(\mathrm{t}_{0.9}\right)$ of up to $8 \mathrm{~s}$ and total time measurements $\left(t_{t}\right) 9-10 \mathrm{~s}$. The data presented show the possibility of rapid determination of hydrogen sulfide by developed sensors.

\subsection{Calibration Characteristic of a Semiconductor Hydrogen Sulfide Sensor}

The dependence of the electrical conductivity of the gassensitive layer of the semiconductor sensor on the content of the detected impurity, as a rule, is nonlinear. Due to the nonlinear nature of the dependence of the conductivity of the sensor on the concentration of the detected substance, it is not very informative. A study of the change in the conductivity of the sensor during chemisorption of various concentrations of hydrogen sulfide was carried out on the measuring setup shown in Figure 1.

The dependence of the useful analytical signal SC- $\mathrm{H}_{2} \mathrm{~S}$ on the concentration of hydrogen sulfide was established in a wide range of its concentrations by passing through a developed sensor a gas mixture of hydrogen sulfide in air. In the experiments performed, each test point in the measuring range was characterized by six values: three for the forward and three for the inverse measurement cycles.

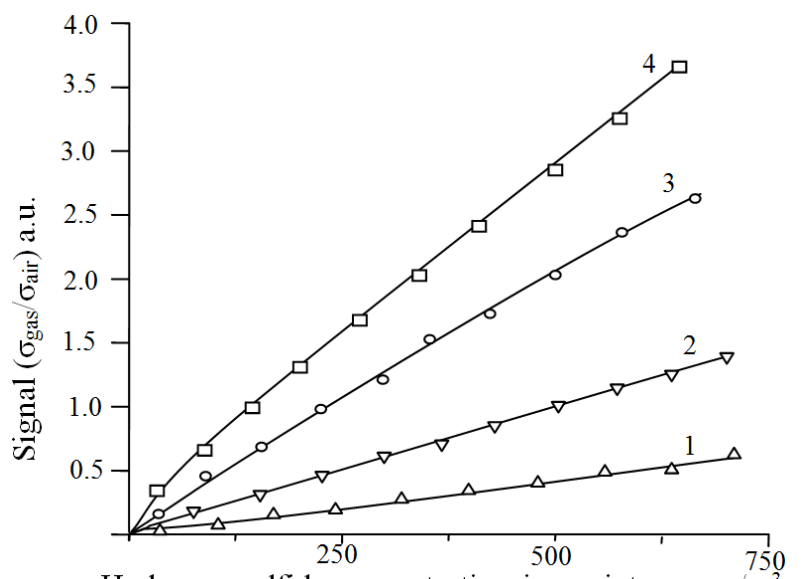

Hydrogen sulfide concentration in a mixture, $\mathrm{mg} / \mathrm{m}^{3}$.

Figure 1. Dependence of the analytical signal of the sensor $\left(\Delta \sigma / \sigma_{\text {air }}\right)$ on the amount of the determined component $\left(\mathrm{C}_{\mathrm{H} 2 \mathrm{~S}}\right)$ in a gas mixture. $1-\mathrm{SiO}_{2}-\mathrm{WO}_{3}$; 2- $\mathrm{SiO}_{2}-\mathrm{WO}_{3}-1 \% \mathrm{CuO} ; 3-\mathrm{SiO}_{2}-\mathrm{WO}_{3}-5 \% \mathrm{CuO}$; 4- $\mathrm{SiO}_{2}-\mathrm{WO}_{3}-10 \% \mathrm{CuO}$.

As a result of the experiments, it was found that the dependence of the resistance of the semiconductor sensor on temperature, as a rule, is nonlinear. Practice shows that this type of dependence is not entirely convenient for basic research in the manufacture and testing of the sensor and gas analyzer. It is more convenient to present the sensor conductivity in a normalized form or in logarithmic coordinates.

In Figure 1 shows the normalized calibration characteristic 
of hydrogen sulfide sensors based on tungsten and copper, with an operating temperature of $350^{\circ} \mathrm{C}$.

As follows from the data presented, in a wide range $(50-$ $750 \mathrm{mg} / \mathrm{m}^{3}$ ), the dependence of the signal of the $\mathrm{SC}-\mathrm{H}_{2} \mathrm{~S}$ semiconductor sensor on the concentration of hydrogen sulfide in the TGM is straightforward.

As a result of the work performed, a method is proposed for increasing the sensitivity of a semiconductor hydrogen sulfide sensor based on the use of a gas-sensitive material based on $\mathrm{SiO}_{2}-\mathrm{WO}_{3}-10 \% \mathrm{CuO}$.

It was found that in the ranges of concentrations of hydrogen sulfide $50-750 \mathrm{mg} / \mathrm{m}^{3}$, the dependence of the signal on the amount of hydrogen sulfide is straightforward. The found values of the basic, absolute, and reduced errors of the developed sensors are much smaller than their permissible values according to GOST.

Table 2. Results of the dependence of the "SC- $\mathrm{H}_{2} \mathrm{~S}$ " sensor signal with a measurement range of $0-1000 \mathrm{mg} / \mathrm{m}^{3}$ on the concentration of hydrogen sulfide in the mixture.

\begin{tabular}{llll}
\hline \multirow{2}{*}{$\begin{array}{l}\text { Given hydrogen sulfide, } \\
\mathbf{m g} / \mathbf{m}^{\mathbf{3}}\end{array}$} & \multicolumn{3}{l}{ Found hydrogen sulfide, $\mathbf{~} \mathbf{g} / \mathbf{m}^{\mathbf{3}}$} \\
\cline { 2 - 4 } & $\overline{x^{ \pm} \Delta \mathbf{x}}$ & $\mathbf{S}$ & $\mathbf{S r} \cdot \mathbf{1 0}^{\mathbf{2}}$ \\
\hline 50 & $51 \pm 2$ & 1.61 & 2.15 \\
100 & $98 \pm 2$ & 1.61 & 1.64 \\
250 & $249 \pm 3$ & 2.41 & 0.67 \\
500 & $496 \pm 4$ & 3.22 & 0.65 \\
750 & $757 \pm 5$ & 4.02 & 0.53 \\
1000 & $992 \pm 5$ & 4.02 & 0.41 \\
\hline
\end{tabular}

Therefore, the developed sensors can be used to monitor the hydrogen sulfide content in objects of various environments.

Checking the measuring range and the basic errors of the
"SC- $\mathrm{H}_{2} \mathrm{~S}$ " sensor. The measurement range of the hydrogen sulfide sensors was checked by supplying a mixture of $\mathrm{H}_{2} \mathrm{~S}$ with air to its input in a certain sequence. The experiments were repeated at least 5 times, the results are presented in table 2.

As follows from the data given in table 2, the developed sensor in the studied concentration range has a direct proportional dependence of the signal on the amount of hydrogen sulfide in the mixture. In the concentration range of $50-1000 \mathrm{mg} / \mathrm{m}^{3}$, the $\mathrm{Sr}$ value does not exceed 0.021 . When determining the basic error of the sensors, a mixture with a certified concentration of hydrogen sulfide vapors in air, which was supplied to the device at a rate of $20-21 / \mathrm{h}$, was used as an TGM.

The main error $(\Delta)$ was determined by the difference between the sensor readings and the true concentration values, referred to the measurement range according to the formula:

$$
\Delta=\mathrm{A}_{\mathrm{i}}-\mathrm{A}_{0}
$$

where $\mathrm{A}_{\mathrm{i}}$ - is the concentration of the measured component at the test point of measurements induced on the indicator; $A_{0}$ is the true concentration of the measured component at the test point.

The main reduced errors ( $p r)$ of the sensors were determined by the formula:

$$
\mathrm{pr}=\left(\mathrm{A}_{1}-\mathrm{A}_{0}\right) * 100 / \mathrm{C}_{\mathrm{f}}-\mathrm{C}_{\mathrm{i}}
$$

where $\mathrm{C}_{\mathrm{i}}$ and $\mathrm{C}_{\mathrm{f}}$ is the initial and final limits of the measured concentration of the determined components of the gaseous media $\left(1000 \mathrm{mg} / \mathrm{m}^{3}\right)$.

\begin{tabular}{|c|c|c|c|c|c|}
\hline \multirow{2}{*}{ No } & \multirow{2}{*}{$\begin{array}{l}\text { Given } \mathrm{H}_{2} \mathrm{~S}, \\
\mathrm{mg} / \mathrm{m}^{3}\end{array}$} & \multirow{2}{*}{$\begin{array}{l}\text { Found hydrogen } \\
\text { sulfide, } \mathrm{mg} / \mathrm{m}^{3}\end{array}$} & \multicolumn{2}{|l|}{ Found error values } & \multirow{2}{*}{$\begin{array}{l}\text { Permissible error in accordance } \\
\text { with GOST }(\mathrm{M}) \%\end{array}$} \\
\hline & & & Main absolute error. $(\Delta)$ & Main error. (؟), \% & \\
\hline 1 & 50 & 51 & 1 & 0.1 & \pm 5.0 \\
\hline 2 & 250 & 247 & 3 & 0.3 & \pm 5.0 \\
\hline 3 & 500 & 496 & 4 & 0.4 & \pm 5.0 \\
\hline 3 & 1000 & 993 & 7 & 0.7 & \pm 5.0 \\
\hline
\end{tabular}

Table 3. The results of determining the basic error of the "SC- $\mathrm{H}_{2} \mathrm{~S}$ " sensor in the range of measured concentrations $0-1000 \mathrm{mg} / \mathrm{m}_{3}(n=5, P=0.95)$.

As follows from the data given in table 4, the found error values of the developed sensors are much less than the permissible values according to GOST. Therefore, the developed sensors can be used to monitor the content of hydrogen sulfide in objects of various environment. The additional error of the "SC- $\mathrm{H}_{2} \mathrm{~S}$ " sensors, caused by a change in the ambient temperature, was checked in the range of -10 $+50^{\circ} \mathrm{C}$. In the experiments, TGM was used with a hydrogen sulfide content of 50 and $500 \mathrm{mg} / \mathrm{m}^{3}$ in a mixture.

The influence of the temperature of the gaseous medium for each point on the additional error of the sensor was determined by the formula:

$$
\mathrm{ad}^{=} \text {main }^{-} \text {norm }
$$

where norm-main error according to the calibration characteristic; main - main sensor error for each measurement.

The results of determining the additional error of the sensor due to a change in ambient temperature showed that the additional error of the $\mathrm{SC}-\mathrm{H}_{2} \mathrm{~S}$ sensor from changes in the temperature of the gas medium is not more than $1.0 \%$, and in all cases it is much less than the main error of the device itself.

\subsection{Selectivity of a Semiconductor Hydrogen Sulfide Sensor}

The most important characteristic of gas analytical instruments is the selectivity of determining the component of the analyzed gas mixture.

In the semiconductor sensors developed by us, the selectivity of determination is ensured by the selection of optimal temperatures and the corresponding catalysts. The selectivity of the determination of hydrogen sulfide by the developed sensors was studied using certified gas mixtures at the request of GOST for gas analytical devices for closed ecological systems and chemical industry facilities. The 
selectivity of the semiconductor hydrogen sulfide sensor was determined in the presence of hydrogen, carbon monoxide and methane. The experiments were carried out at a temperature of $\mathrm{SC}-\mathrm{H}_{2} \mathrm{~S}$ using standard gas mixtures, the composition of which is shown in table 4 .

Table 4. Composition and parameters of calibration gas mixtures used to determine the selectivity of the hydrogen sulfide sensor.

\begin{tabular}{|c|c|c|c|c|c|}
\hline \multirow{2}{*}{ No } & \multirow{2}{*}{ Content of TGM } & \multicolumn{4}{|c|}{ Component content, $\mathrm{mg} / \mathrm{m}^{3}$} \\
\hline & & $\mathrm{H}_{2} \mathrm{~S}$ & $\mathbf{H}_{2}$ & $\mathrm{CO}$ & $\mathrm{CH}_{4}$ \\
\hline 1 & $\mathrm{H}_{2} \mathrm{~S}+$ air & $356.0 \pm 0.6$ & - & - & - \\
\hline 2 & $\mathrm{H}_{2} \mathrm{~S}+\mathrm{H}_{2}+$ air & $356.0 \pm 1.0$ & $460.0 \pm 1.8$ & - & - \\
\hline 3 & $\mathrm{H}_{2} \mathrm{~S}+\mathrm{CO}+$ air & $356.0 \pm 0.8$ & - & $380.0 \pm 2.5$ & - \\
\hline 4 & $\mathrm{H}_{2} \mathrm{~S}+\mathrm{CH}_{4}+$ air & $356.0 \pm 1.0$ & - & - & $450.0 \pm 1.5$ \\
\hline
\end{tabular}

The experiments were carried out at a temperature of $20 \pm 2^{\circ} \mathrm{C}$ and a pressure of $730 \pm 10 \mathrm{~mm} \mathrm{Hg}$. A mixture of No 1 (hydrogen sulfide + air) was supplied to the PPS input for 5 min, the readings were recorded with a digital voltmeter, then a mixture of $\mathrm{N}_{2}$ (hydrogen sulfide + hydrogen + air) was fed, and after 5 minutes the readings of the digital voltmeter were re-recorded.

Table 5. Selectivity results for a semiconductor hydrogen sulfide sensor $S C-H_{2} S-3 M(n=5, P=0.95)$.

\begin{tabular}{|c|c|c|c|c|c|c|}
\hline \multirow{3}{*}{$\begin{array}{l}\text { The composition of the gas } \\
\text { mixture, } \mathrm{mg} / \mathrm{m}^{3} \text {. }\end{array}$} & \multicolumn{6}{|c|}{ Found hydrogen sulfide, $\mathrm{mg} / \mathrm{m}^{3}$. } \\
\hline & \multicolumn{2}{|c|}{ SC $\mathrm{H}_{2} \mathrm{~S}-$ № 1} & \multicolumn{2}{|c|}{$\mathrm{SC} \mathrm{H}_{2} \mathrm{~S}-$ № 2} & \multicolumn{2}{|c|}{$\mathrm{SC} \mathrm{H}_{2} \mathrm{~S}-$ № 3} \\
\hline & $\bar{x} \pm \Delta \mathbf{X}$ & $\mathrm{Sr}^{*} 10^{2}$ & $\bar{x} \pm \Delta \mathbf{X}$ & $\mathrm{Sr}^{*} 10^{2}$ & $\bar{x} \pm \Delta \mathbf{X}$ & $\mathrm{Sr}^{* 10^{2}}$ \\
\hline $\mathrm{H}_{2} \mathrm{~S}-56.0+$ air. & $354.0 \pm 3.2$ & 1.1 & $351.0 \pm 1.5$ & 0.8 & $355.0 \pm 2.2$ & 1.0 \\
\hline $\mathrm{H}_{2} \mathrm{~S}-356.0+\mathrm{H}_{2}-460.0+$ air & $355.0 \pm 4.2$ & 1.0 & $350.0 \pm 2.8$ & 0.6 & $357.0 \pm 4.1$ & 0.9 \\
\hline $\mathrm{H}_{2} \mathrm{~S}-356.0+\mathrm{CO}-380.0+$ air & $352.0 \pm 2.5$ & 0.7 & $358.0 \pm 3.0$ & 0.8 & $357.0 \pm 2.9$ & 0.8 \\
\hline $\mathrm{H}_{2} \mathrm{~S}-356.0+\mathrm{CH}_{4}-450.0+$ air & $356.0 \pm 3.9$ & 0.9 & $359.0 \pm 4.6$ & 1.0 & $359.0 \pm 4.3$ & 1.0 \\
\hline
\end{tabular}

Similarly, signals were obtained for a mixture of $\mathrm{N} 3$ (hydrogen sulfide + carbon monoxide + air) and $\mathrm{N} 4$ (hydrogen sulfide + methane + air). The number of parallel measurements for each standard gas mixture is 5 . The average results obtained when establishing the selectivity of $\mathrm{SC}-\mathrm{H}_{2} \mathrm{~S}$ are presented in Table 5 .

As follows from the obtained experimental data, the presence of carbon monoxide (up to $380 \mathrm{mg} / \mathrm{m}^{3}$ ), hydrogen (up to $460 \mathrm{mg} / \mathrm{m}^{3}$ ) and methane (up to $450 \mathrm{mg} / \mathrm{m}^{3}$ ) in the analyzed mixture $\left(\mathrm{H}_{2} \mathrm{~S}+\right.$ air $)$ does not affect the value of the sensor output signal hydrogen sulfide. From the above data (Table 5), it follows that the developed sensor in the studied range of $\mathrm{H}_{2} \mathrm{~S}$ concentrations allows its selective determination. The error in determining $\mathrm{SC}-\mathrm{H}_{2} \mathrm{~S}$ due to unchanged components does not exceed $1.5 \%$.

Thus, a sensor has been developed that provides selectivity for the determination of hydrogen sulfide in multicomponent gas-air mixtures, which simultaneously contain hydrogen sulfide, carbon monoxide and methane (natural methane gas). Such mixtures include gaseous emissions from industrial enterprises producing sulphides of metals, paints, artificial silk, sugar, asphalt, leather products, atmospheric air from mines, livestock farms, sewers, wells, etc. In all cases, the value of the relative standard deviation $(\mathrm{Sr})$ for the account of unmeasured components does not exceed 0.05 .

\subsection{Stability of a Semiconductor Hydrogen Sulfide Sensor}

One of the main indicators of sensors is the signal stability over time. In order to establish the possibility of practical application of a sensor of hydrogen sulfide concentration in a production environment, a study was conducted of its stability under prolonged exposure to hydrogen sulfide. In the research process, a gas-air mixture with a concentration of hydrogen sulfide of $50 \mathrm{mg} / \mathrm{m}^{3}$ was used.

The operating temperature of the sensor was $350^{\circ} \mathrm{C}$. During the research, an artificial climate chamber was used. The chamber has the ability to maintain air humidity from existing in the environment to $90 \%$ at a temperature of 10 to $+60^{\circ} \mathrm{C}$. Regulation and maintenance of air temperature is carried out automatically using electronic bridges. The accuracy of maintaining the set temperature is $0.1^{\circ} \mathrm{C}$. The stability of the sensor was determined as a result of an experiment conducted for 30 days ( 720 hours) by exposure to $\mathrm{H}_{2} \mathrm{~S}$-containing gas for 24 hours daily on a sensor based on $\mathrm{SiO}_{2} / \mathrm{WO}_{3}+10 \% \mathrm{CuO}$.

The measurement of the input signal during a regulated time was recorded on a chart tape of a recording device while recording the temperature and pressure of the environment. When processing the test results, random single discharge of the output signal were not taken into account for a duration of each emission of not more than $10 \mathrm{~s}$. and the amount of discharge of not more than three during each day of operation of the semiconductor sensor. The results of the 720-hour experiment are presented in table. 6, from which it follows that the output signal $\mathrm{SC}-\mathrm{H}_{2} \mathrm{~S}$ for a regulated time is stored stable.

The change in the value of the output signal for a regulated time was estimated by the maximum divergence of the sensor signal:

$$
\Delta \operatorname{tg}=\left(U p_{\max }-U p_{\min }\right) 100 / U_{s c}
$$

where $\Delta \operatorname{tg}$ is the limit of the permissible change in the output signal for a regulated time interval; $\mathrm{Up}_{\max }$, and $\mathrm{Up}_{\min }$ maximum and minimum signal discrepancies; $U_{s c}$ - scale of the device (KSP 0-1000 kOhm).

Our calculations show that the value of $\Delta \operatorname{tg}$ for a regulated 
time interval is $2.3 \%$.

Table 6. Signal stability of a semiconductor sensor with gas-sensitive material $\mathrm{SiO}_{2} / \mathrm{WO}_{3}+10 \% \mathrm{CuO}\left(\mathrm{C}_{\mathrm{NH} 3}=50,0 \mathrm{mg} / \mathrm{m}^{3}, n=5, \mathrm{P}=0.95\right)$.

\begin{tabular}{llllll}
\hline \multirow{2}{*}{$\begin{array}{l}\text { Time } \\
\text { hour }\end{array}$} & \multicolumn{2}{c}{ Environmental parameter value } & \multicolumn{3}{c}{ Sensor signal, kOhm } \\
\cline { 2 - 6 } & temperature, ${ }^{\circ} \mathbf{C}$ & $\begin{array}{l}\text { Pressure, } \mathbf{~ m m} \\
\mathbf{H g}\end{array}$ & $\bar{x} \pm \mathbf{\Delta} \mathbf{X}$ & $\mathbf{S}$ & \multirow{2}{*}{$\mathbf{S r}^{*} \mathbf{1 0}^{\mathbf{2}}$} \\
\hline 1 & 20.5 & 723 & $51 \pm 0.5$ & 0.42 & \\
5 & 20.0 & 730 & $50 \pm 0.7$ & 0.33 & \\
10 & 20.9 & 733 & $51 \pm 0.4$ & 0.32 & \\
25 & 20.6 & 730 & $47 \pm 0.3$ & 0.24 & \\
50 & 20.7 & 726 & $47 \pm 0.5$ & 0.40 \\
100 & 20.5 & 742 & $50 \pm 0.6$ & 0.28 \\
200 & 20.0 & 730 & $51 \pm 0.6$ & 0.48 \\
300 & 20.4 & 732 & $48 \pm 0.4$ & 0.32 \\
400 & 20.5 & 736 & $50 \pm 0.5$ & 0.40 \\
500 & 20.6 & 739 & $52 \pm 0.6$ & 0.48 & \\
600 & 20.5 & 741 & $52 \pm 0.4$ & 0.32 \\
700 & 20.8 & 736 & $50 \pm 0.6$ & 0.48 \\
720 & 20.5 & 740 & $48 \pm 0.5$ & 0.40 \\
\hline
\end{tabular}

As a result of the 720-hour experiment, the resistance of the semiconductor sensor increased by $2.5 \%$, and the sensitivity decreased by $1.8 \%$. Such a drift of the sensor parameters introduces a slight error in the measurement, but satisfies the production requirements.

Change in gas flow rate in the studied interval $(5-50 \mathrm{l} / \mathrm{h})$ does not significantly affect the value of the sensor output. The output signal of the sensors also does not depend on the location in space and the angles of inclination, which allows us to classify the developed sensors (according to GOST13320-82) as independent.

\subsection{The Effect of Pressure on the Values of the Input Signals $\mathrm{SC}-\mathrm{H}_{2} \mathrm{~S}$}

Pressure tests were carried out in the range of $600-900 \mathrm{~mm}$ $\mathrm{Hg}$. To determine the effect of pressure on the operation of the sensor, the pressure in the sensor chamber was set from 600 to $900 \mathrm{~mm} \mathrm{Hg}$. (with a difference of 50.0-1.5 mm Hg) and after one hour the readings of the device were recorded while passing a standard mixture with a hydrogen sulfide content of 250 and $500 \mathrm{mg} / \mathrm{m}^{3}$.

The results of the experiments to determine the effect of pressure on the useful analytical signal are presented in table 7.

As follows from the above data, in the studied range, a change in the pressure of the gaseous medium has practically no effect on the value of the sensor output signal.
Thus, as a result of the experiments, a selective semiconductor hydrogen sulfide sensor was developed, which provides rapid determination of hydrogen sulfide in a wide range of its concentration in atmospheric air and process gases. The developed semiconductor hydrogen sulfide sensors are not inferior in accuracy and reproducibility to the known foreign analogues, while maintaining the following characteristics: expressness, portability, ease of manufacture and operation. The value of the relative standard deviation $(\mathrm{Sr})$ due to unchanged components does not exceed 0.05 . Changing the flow rate of the gas mixture in the studied interval $(5-50 \mathrm{l} / \mathrm{h})$ does not significantly affect the value of the sensor output signal. The output signal of the sensors also does not depend on the location in space and the angles of inclination, which allows us to classify the developed sensors (according to GOST13320-82) as independent.

Table 7. The results of determining the concentration of hydrogen sulfide at various pressures $(n=5, P=0.95)$.

\begin{tabular}{|c|c|c|c|c|}
\hline \multirow{3}{*}{$\begin{array}{l}\text { Pressure of gas } \\
\text { mixture, } \mathrm{mm} \mathrm{Hg}\end{array}$} & \multicolumn{4}{|c|}{ Sensor signal, $\left(\Delta \sigma / \sigma_{\text {air }}\right)$, a. u. } \\
\hline & \multicolumn{2}{|c|}{$\mathrm{C}_{\mathrm{H} 2 \mathrm{~s}}-250 \mathrm{mg} / \mathrm{m}^{3}$} & \multicolumn{2}{|c|}{$\mathrm{C}_{\mathrm{H} 2 \mathrm{~S}}-500 \mathrm{mg} / \mathrm{m}^{3}$} \\
\hline & $\bar{x} \pm \Delta \mathbf{x}$ & $\mathrm{Sr} \cdot 10^{2}$ & $\bar{x} \pm \Delta \mathbf{x}$ & $\mathrm{Sr} \cdot 10^{2}$ \\
\hline 600 & $2.57 \pm 0.02$ & 0.88 & $3.26 \pm 0.9$ & 0.69 \\
\hline 700 & $2.58 \pm 0.02$ & 0.86 & $3.28 \pm 1.7$ & 1.32 \\
\hline 800 & $2.57 \pm 0.02$ & 0.89 & $3.27 \pm 1.8$ & 1.40 \\
\hline 900 & $2.59 \pm 0.03$ & 1.32 & $3.26 \pm 0.9$ & 0.71 \\
\hline
\end{tabular}

\subsection{Resistance of the Device to Concentration Overloads}

The stability test of the hydrogen sulfide sensor to overloads of its concentration was carried out with a hydrogen sulfide content of $2500 \mathrm{mg} / \mathrm{m}^{3}$ in the mixture. As a control mixture, a gas mixture was used, where the hydrogen sulfide content was $500 \mathrm{mg} / \mathrm{m}^{3}$, at this concentration the output signals of "SC- $\mathrm{H}_{2} \mathrm{~S}$ " were compared before and after exposure to an overload mixture. The exposure time of the transshipment mixture is 20 minutes

The recovery time of the normal operation of the " $\mathrm{SC}-\mathrm{H}_{2} \mathrm{~S}$ " sensor was determined by its output signal in the zone of the main error. The results of the experiments showed that the developed hydrogen sulfide sensor in the studied concentration range withstands overloads under the tested parameters (Table 8).

Table 8. The results of testing the stability of " $\mathrm{SC}-\mathrm{H}_{2} \mathrm{~S}$ " to overloads by Concentrations $(n=5, P=0.95)$.

\begin{tabular}{|c|c|c|c|c|c|}
\hline \multirow[t]{2}{*}{ No } & \multirow{2}{*}{$\begin{array}{l}\text { The concentration of hydrogen } \\
\text { sulfide in the mixture. }\end{array}$} & \multicolumn{2}{|c|}{$\begin{array}{l}\text { Sensor output before and after air overload, } \\
\mathrm{mg} / \mathrm{m}^{3}(\mathrm{vol} . \%)\end{array}$} & \multirow{2}{*}{$\begin{array}{l}\text { The main } \\
\text { absolute error }\end{array}$} & \multirow{2}{*}{$\begin{array}{l}\text { Tolerance the meaning of } \\
\text { the main error in } \\
\text { accordance with GOST }\end{array}$} \\
\hline & & before air overload & after air overload & & \\
\hline 1 & $\mathrm{C}_{\mathrm{H} 2 \mathrm{~S}}=500 \mathrm{mg} / \mathrm{m}^{3}$ & $506 \pm 5$ & $512 \pm 5$ & 6 & $\pm 0.25 \%$ \\
\hline 2 & $\mathrm{C}_{\mathrm{H} 2 \mathrm{~S}}=2500 \mathrm{mg} / \mathrm{m}^{3}$ & $2497 \pm 9$ & $2504 \pm 6$ & 7 & $\pm 0.25 \%$ \\
\hline
\end{tabular}

The value of the additional error " $\mathrm{SC}-\mathrm{H}_{2} \mathrm{~S}$ " under the influence of overload concentration on the operation of the device does not exceed 0.03 vol.\%. Thus, our results made it possible to develop a selective semiconductor hydrogen sulfide sensor "SC- $\mathrm{H}_{2} \mathrm{~S}$ " designed to automatically monitor the concentration of hydrogen sulfide in gas samples.
Assessment of the metrological characteristics of the developed "SC- $\mathrm{H}_{2} \mathrm{~S}$ " sensor was carried out in accordance with GOST 52033-2003. During the experiment, the following were determined: measurement ranges; basic absolute, relative, reduced and additional errors when changing environmental parameters (temperature, pressure, 
humidity, tilt angle, traffic shocks, mechanical shocks, etc.). It was found that in the ranges of concentration of hydrogen sulfide $50-1000 \mathrm{mg} / \mathrm{m}^{3}$ the dependence of the signal on the amount of hydrogen sulfide is straightforward. The found values of the basic absolute and reduced errors of the developed sensors are much smaller than their permissible values according to GOST. Therefore, the developed sensors can be used to monitor the hydrogen sulfide content in objects of various media. The results of determining the additional error of the sensor due to a change in ambient temperature, equal to $0.7 \%$ and in all cases much less than the main error of the device itself. The total additional error of the sensor due to changes in the temperature and pressure of the gas medium in all cases was no more than $\pm 1.5 \%$. The stability of the hydrogen sulfide sensor to overloads of the concentration of hydrogen sulfide was tested at a hydrogen sulfide content of $2500 \mathrm{mg} / \mathrm{m}^{3}$ in the mixture.

The experiments showed that the developed hydrogen sulfide sensor in the studied concentration range withstands concentration overloads, and its signal in the studied time interval remains stable over the entire range of changes in the concentration of hydrogen sulfide in the air.

As a result of the work carried out, a method is proposed for increasing the selectivity and sensitivity of semiconductor methods for determining hydrogen sulfide, based on the use of optimal values of the sensor temperature and the composition of the gas-sensitive material of the catalysts, which have inadequate activity for different components of the gas mixture. The developed semiconductor hydrogen sulfide sensors are not inferior in accuracy and reproducibility to the known foreign analogues, while maintaining the following characteristics: expressness, portability, ease of operation and manufacture. The value of the relative standard deviation $(\mathrm{Sr})$ due to unmeasured components does not exceed 0.05. Changing the flow rate of the gas mixture in the studied interval $(5-50 \mathrm{l} / \mathrm{h})$ does not significantly affect the value of the sensor output signal and its location in space and the tilt angles, which allows us to classify the developed sensors (according to GOST13320-82) as independent.

The semiconductor hydrogen sulfide sensors developed in this work are used:

i. In environmental monitoring systems;

ii. In devices for monitoring leaks from gas apparatus and equipment;

iii.In systems for ensuring the safety of work and signaling to explosive concentrations of hydrogen sulfide;

iv. In control systems of continuous technological processes associated with a change in the gas environment

Based on the results of the work performed in paper, the following conclusions can be made.

\section{Conclusion}

Semiconductor sensors have been developed for monitoring hydrogen sulfide in atmospheric air and process gases, which allow selective monitoring of the hydrogen sulfide content in the presence of combustible gases $(\mathrm{CO}$, hydrogen, and methane) in wide ranges of their concentrations.

The use of the developed sensors significantly reduces the analysis error, increases the lifetime and stability of the device, while improving the reproducibility and selectivity of hydrogen sulfide determination.

The main metrological characteristics and operational parameters of the developed selective semiconductor sensors for determining hydrogen sulfide are studied. The selectivity, the measurement range and the main error, the additional error of the sensor due to changes in ambient temperature, the effect of pressure on the values of the input signals SC$\mathrm{H}_{2} \mathrm{~S}$, and the instrument's resistance to concentration overloads are established.

The $\mathrm{SC}-\mathrm{H}_{2} \mathrm{~S}$ developed in terms of accuracy and reproducibility is in no way inferior to the well-known foreign analogues, while maintaining the following characteristics: expressness, portability, ease of operation and manufacture. The value of the relative standard deviation $(\mathrm{Sr})$ due to unmeasured components does not exceed 0.05 . Changing the flow rate of the gas mixture in the studied interval $(5-50 \mathrm{l} / \mathrm{h})$ does not significantly affect the value of the sensor output signal and its location in space and the tilt angles, which allows us to classify the developed sensors (according to GOST-13320-82) as independent. The scope of the developed sensors: environmental monitoring of atmospheric air and process gases.

\section{References}

[1] Abdurakhmanov B. M. Thermocatalytic automatic determination of hydrogen sulfide in air and process gases / Abstract of dissertation for the degree of candidate of chemical sciences. Tashkent - 2009. 24 p.

[2] Moskvin L. N., Papsueva A. G., Shimarygin R. N. Continuous determination of hydrogen sulfide and sulfur-containing organic impurities in atmospheric air // Abstracts 5 AllRussian Conference on the Analysis of Environmental Objects "ECOANALYTICS-2003". St. Petersburg, 2003, p. 248.

[3] Harmful substances in industry. Directory. Under the total. Edited by N. V. Lazarev Volume 3., Ed. "Chemistry", Leningrad branch. 1977. p. 49-75.

[4] Glebova EV, Golubev Yu. D., Prosnurov A. P., Yankovich A. Kh., Kashirskaya L. M. Estimation of air pollution during open sulfur storage // Safe. labor in industry. 1990.- No. 3. -C. 36-37.

[5] Perekrestov A. P. The effect of hydrogen sulfide on the intensity of corrosion-mechanical wear // Herald of mechanical engineering. 2006.- No. 9. -P. 44.

[6] Begmatov Rizamat Template synthesis of gas-sensitive organic-inorganic materials for semiconductor sensors.//Dissertation abstract of doctor of philosophy in chemical sciences. Samarkand-2020. p. 44.

[7] OAO NPF "Geofizika" - Laboratory "Information-measuring systems GTI" http://www.npf-geofizika.ru/leuza/index.html. 
[8] Myasnikov I. A., Sukharev V. Ya., Kupriyanov L. Yu., Zavyalov S. A. Semiconductor sensors in physical and chemical studies // M.: Nauka, 1991. -327s.

[9] Obvintseva L. A. Semiconductor metal oxide sensors for determining chemically active gas impurities in the air // Ros. Chem. g. (J. Ros. Chem. Society named after D. I. Mendeleev), 2008, T. LII, No. 2. - S. 113-121.
[10] Abdurakhmanov I. E., Kabulov B. J. Selection of a selective catalyst for a semiconductor hydrogen sulfide sensor / Acta UzMU. 2016. P. 89-93.

[11] Abdurakhmanov I. E., Kabulov B. J. Catalyst for a semiconductor hydrogen sulfide sensor / Request for an invention RUz. IPC (8) G 01№ 27/16. 